\title{
Modern Imaging in Planning \\ a Personalized Hip Replacement and Evaluating the Spino-pelvic Relationship in Prosthetic Instability
}

\author{
Omar A. Behery, Lazaros Poultsides, \\ and Jonathan M. Vigdorchik
}

\section{Key Points}

- Personalized component implantation in total hip arthroplasty aims to reproduce normal hip joint anatomy and improve functional outcomes and implant survivorship.

- Traditional radiographic evaluation for total hip arthroplasty consists of an anteroposterior view of the pelvis and a cross table lateral of the hip, and is useful to delineate anatomy and component sizing, but does not take into account the dynamic position of the hip joint in different postural positions.

- The conventional acetabular component "safe zone" does not account for the spino-pelvic relationship and the dynamic nature of acetabular component orientation, which impacts the function and stability of a total hip arthroplasty.

O. A. Behery · L. Poultsides

NYU Langone Health, NYU Langone Orthopedic Hospital, New York, NY, USA

e-mail: Omar.Behery@nyulangone.org

J. M. Vigdorchik ( $\square)$

Hospital for Special Surgery, New York, NY, USA

e-mail: VigdorchikJ@HSS.EDU
- Sitting and standing alignment radiographs have gained recent popularity and are important to routinely obtain and analyze to determine the best patient-specific component position, given the high concordance between hip and spine pathology.

- Three-dimensional cross-sectional imaging or 2-D/3-D reconstructions can also be useful to better delineate hip anatomy and template component size and position.

- Postoperatively CT imaging can be useful in assessing the accuracy and quality of personalized total hip component implantation.

\subsection{Introduction}

Successful total hip arthroplasty (THA) greatly depends on appropriate implant choice and accurate femoral and acetabular component positioning. Preoperative radiographic templating is crucial, and accurate intraoperative execution of the templated plan is important to maximize implant stability and bearing performance. Traditionally, plain radiographs have been used for preoperative planning, as well as postoperative follow-up and assessment of component position, with historically defined "safe zones" 
for component position. However, as our understanding of optimal implant positioning in the setting of spino-pelvic dynamics has expanded, more advanced methods of radiographic assessment of implant positioning have gained popularity. Given the variations in anatomy and functional kinematics of a patient's hip joint, the optimal THA component alignment and positioning may differ on a case by case basis, and therefore, advanced methods of assessing optimal patientspecific implant positioning are of prime importance.

\subsection{Personalized Total Hip Arthroplasty}

Personalized techniques for implanting hip components have been developed with the goal to solve residual complications that occur with conventionally implanted hip prostheses. One of the causes of failure in conventionally implanted hip prostheses is the suboptimal interaction between components (e.g., edge loading and prosthetic impingement). This is primarily related to the systematic and generalized approach for templating and implanting total hip components in the traditional technique (similar implants positioning for all patients), thereby disregarding the unique individual joint anatomy, biomechanics, and spino-pelvic dynamics. Personalized techniques for joint replacement have therefore been developed to address these issues and improve on the outcomes of THA. This represents a paradigm shift in the approach to THA.

Personalized techniques for THA aim to reproduce normal hip anatomy and biomechanics to generate a more physiological prosthetic hip to improve function, patient satisfaction, and implant survivorship. The growing knowledge surrounding the impact of spino-pelvic dynamics on the stability of a THA is an important discussion in the delivery of personalized total hip components. A more detailed description of the evolution of hip arthroplasty from traditional systematic to modern patient-specific kinematic techniques can be found in the Chap. 3 (hip replacement: development and future). This para- digm shift in the technique for implanting hip components from a traditional, systematic approach toward personalized component implantation necessitates developing reliable methods of postoperative radiographic evaluation and assessment of the accuracy and quality of personalized hip component implantation.

\subsection{Traditional Radiographic Evaluation}

Traditional radiographic evaluation consists of plain films. An array of different projections can be obtained to gain information regarding hip pathology, alignment, osseous anatomy and morphology, as well as bone quality. Following THA, plain films can demonstrate implant alignment, positioning, the presence of a periprosthetic fracture, as well as reactive bony changes such as osteolysis and stress shielding. Radiographs are typically easy to obtain, less expensive compared with advanced imaging, but may be somewhat limited in providing information on important anatomical relationships such as femoral neck anteversion and functional acetabular orientation.

\subsubsection{Anteroposterior (AP) View of the Pelvis}

This projection is obtained supine or weight bearing, with both legs internally rotated $15^{\circ}$ to obtain a profile view of the femoral neck anatomy which is on average $15^{\circ}$ anteverted. In order to properly assess implant positioning on an AP pelvis, it is important that the image is obtained with the proper technique and with a marker of a known size (typically $25 \mathrm{~mm}$ ) present as close to the hip joint as possible for calibrating size and accurate magnification. The hip center of rotation is the center of the femoral head articulating within the acetabular cup. Leg lengths can be estimated by drawing a horizontal reference line connecting both teardrops (or ischial tuberosities) and comparing the perpendicular distance from that line to a similar reference point on the 
proximal femur, typically the lesser trochanter. On the acetabular side, the static supine or standing cup abduction angle can be measured by using the horizontal reference line connecting both tear drops and measuring the acute angle subtended by an intersecting line connecting the superior and inferior edges of the cup (Fig. 13.1a). The static supine or standing cup anteversion may also be measured on an AP pelvis using one of multiple methods such as the Lewinnek method which is based on a mathematical formula [1] (Fig. 13.1b) or using computer software based on the geometry of the ellipse created by the anterior and posterior lips of the cup. On the femoral side, stem size and fit can be evaluated based on knowledge of the implant and expected fixation pattern. The varus/valgus alignment of the stem can be assessed based on any deviation of the stem from the alignment of the femoral canal, and femoral offset can be measured from the center of rotation of the hip joint to a line traveling down the femoral canal. Furthermore, the static supine or standing femoral version can be estimated based on the AP pelvis radiograph as described by Weber et al. [2]. This technique relies on calculating the femoral version by rotation-based change in the measured neckshaft angle of the stem, using the following formula: Stem version $=\operatorname{arcos}[\tan$ (measured neck shaft angle)/tan (true implant neck shaft angle)]. An alternative technique of measuring femoral

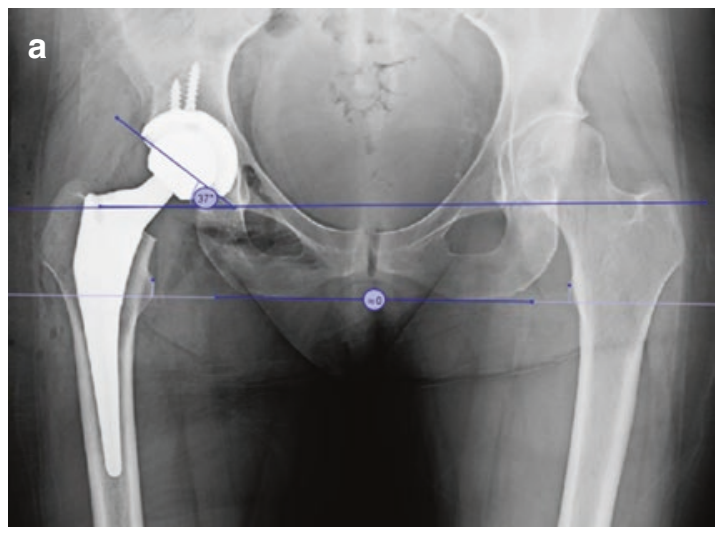

Fig. 13.1 (a) Acetabular component inclination may be estimated on this supine anteroposterior view of the pelvis based on a horizontal reference line connecting the tear version has been described based on a specialized posteroanterior seated hip radiograph called a Budin view [3]. Computed tomography is the gold standard in measuring the anatomic femoral anteversion, which is made relative to the posterior condylar line of the knee.

\subsubsection{Cross Table and Frog-Lateral Views}

A cross table lateral is obtained in the supine position, with the leg internally rotated $15^{\circ}$, contralateral hip flexed, with the beam centered over the femoral head and aimed $45^{\circ}$ in the coronal plane to avoid the contralateral hip. On this projection, the static supine acetabular anteversion can be measured by the angle created between a line over the face of the cup and a line that is perpendicular to the horizontal plane as described by Woo and Morrey. This measurement however is prone to inaccuracy as it can be affected by pelvic tilt, which changes as the contralateral hip is flexed. A more recent employment is the ischio-lateral method of estimating anteversion is based off of the longitudinal axis of the ischial tuberosity and can avoid this issue [4]. The femoral stem fit and anteroposterior angulation is also visualized on this view, but the proximal femur is better visualized on a frog-lateral radiograph, which is obtained by centering the beam

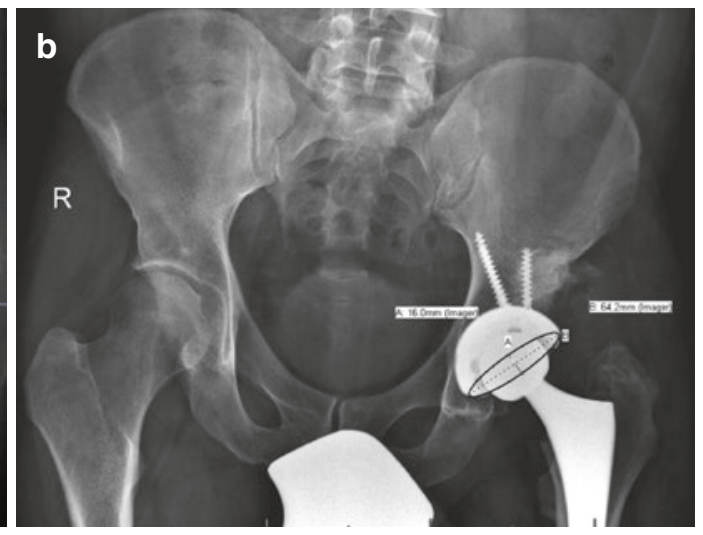

drops. (b) Acetabular component anteversion calculated based on Lewinnek's method (Version $=$ Arcsin (short axis/long axis)) to be approximately $25^{\circ}$ 
over the femoral head with the hip flexed and abducted $45^{\circ}$. Although this view is a lateral of the proximal femur, it is not a lateral view of the acetabular cup.

\subsubsection{Shortcomings of Traditional Radiographic Assessment}

There are some important considerations that are not completely evaluated using the traditional radiographic methods. For instance, plain films are two-dimensional, and an AP view of the pelvis only allows for coronal plane templating of the acetabular component. The thickness and width of the anterior and posterior walls are not visualized, and therefore unaccounted for when templating acetabular component size. Although knowing femoral head diameter may reproducibly allow deduction of a reliable cup size template, axial imaging may better visualize acetabular anterior and posterior wall bone stock and therefore more accurate component size templating.

Furthermore, plain radiographic assessment only provides static landmarks of acetabular inclination and anteversion, which assumes a constant position of the acetabulum. Changes in acetabular inclination and anteversion secondary to postural pelvic obliquity, tilt, or rotation in a weight bearing position may be completely missed on AP pelvis views (supine or standing). Static imaging also ignores the dynamic relationships between the acetabular position, the pelvis, and the spine, which change in different postural positions. Patients may have physiologically or pathologically different profiles of spino-pelvic mobility which can impact cup position and therefore their risks of instability, prosthetic impingement, and edge loading if these variables are ignored by using a universally defined "safe zone" target of cup position of $40 \pm 10^{\circ}$ of inclination and $15 \pm 10^{\circ}$ of anteversion as defined by Lewinnek [1]. In fact, in a large cohort of 9784 patients, 58\% of THA dislocations occurred in patients with components placed in the classically defined "safe zone" [5].

Traditional plain radiography may be inadequate in judging the quality of personalized total hip component implantation. Postoperative radiographs have been shown to lack precision when assessing the quality of the restoration of the hip biomechanical parameters (femoral medial offset and femoral length) and cannot fully inform if the personalized implants have been positioned to reproduce the native hip anatomy and match the individual spino-pelvic dynamics. For instance, plain films do not inform the operator if the cup is oriented parallel to the native transverse acetabular ligament, nor if the adjustment of anteversion to accommodate a stiff lumbar spine has been precisely achieved, or whether the prosthetic neck anteversion has reproduced the native femoral anteversion. These limitations of static, 2-D plain radiographs in the postoperative evaluation of personalized component positioning compel the use of more advanced imaging techniques.

\subsection{Modern Concepts and Radiographic Evaluation}

The dynamic relationship between the pelvis and the lumbar spine affects acetabular cup position and can therefore profoundly impact the stability of THA. Hip pathology frequently coexists with lumbar spine pathology, and lumbar stiffness or fusion has been linked with increased instability following THA $[6,7]$. This warrants thorough radiographic assessment and analysis of spinopelvic parameters and determination of spinopelvic motion when preoperatively planning the ideal acetabular implant and cup position, to estimate a "safe zone" that is specific to the patient evaluated. Traditionally, the transverse acetabular ligament has been used to guide 
patient-specific cup anteversion; however, given the dynamic nature of the hip joint, the functional anteversion of the acetabulum may differ based on pelvic tilt [8].

\subsubsection{Sitting and Standing Alignment Radiographs}

Although not routinely obtained, sitting and standing lateral full-length radiographs are often obtained to determine the changes in spino-pelvic parameters and become especially important to obtain in patients with lumbar spinal disease or fusion or to evaluate acetabular component position if presenting with recurrent total hip instability $[9,10]$. It is known that patients with a stiff or fused spine, who experience prosthetic dislocation, have a tendency to demonstrate decreased spine flexion, smaller change in pelvic tilt, and increased hip flexion from standing to sitting position [11]. These sitting and standing films may be obtained on a 36-inch film cassette or if available, using EOS $^{\mathrm{TM}}$ stereoradiographs (EOS ${ }^{\mathrm{TM}}$ Imaging, Paris, France) (Fig. 13.2a-d). More dynamic imaging including flexed-seated and single-leg step-up lateral images are gaining popularity as they may be better at assessing the functional position of the hip joint and spinopelvic dynamics and have been used for an Optimized Positioning System ${ }^{\mathrm{TM}}$ used to preoperatively plan patient-specific target component position [12].

Several spino-pelvic parameters can be measured and analyzed on the lateral sitting and standing alignment films (Fig. 13.3):

(a) Pelvic tilt (PT) or pelvic version may be measured as the angle between the vertical axis and a line connecting the center of the $\mathrm{S} 1$ vertebral endplate and the center of the femoral head. Pelvic tilt increases as the pelvis retroverts when going from standing to a sitting position. (b) Sacral slope (SS) can be measured as the angle between a horizontal reference line and a line parallel to the $\mathrm{S} 1$ endplate. This parameter decreases as the pelvis goes into retroversion.

(c) Pelvic incidence is the sum of SS and PT and can be measured as the angle between a line connecting the femoral head and the center of the S1 endplate and a line perpendicular to the $\mathrm{S} 1$ endplate. This parameter remains constant through pelvic motion; however, it can be used as a direct indicator of the ability to recruit pelvic tilt to compensate for spinal deformity.

(d) Lumbar lordosis (LL) is the Cobb angle between two lines parallel to the L1 and the $\mathrm{S} 1$ endplates. This value is typically within $10^{\circ}$ of the PI in a normal lumbar spine.

(e) The anterior pelvic plane (APP) can be used to measure pelvic tilt as well. It is created by a line connecting both anterior superior iliac spines and the pubic symphysis, and the angle created between this plane and the vertical axis represents that anterior pelvic plane-pelvic tilt (APP-PT) angle.

In a normal and flexible lumbar spine, the pelvic tilt increases when going from standing to sitting, which increases acetabular anteversion and decreases the risk of impingement and posterior dislocation. Acetabular anteversion increases by $0.7^{\circ}$ for each $1^{\circ}$ increase in pelvic tilt [13]. However, in the case of a stiff or fused lumbar spine, the change in pelvic tilt markedly decreases from standing to sitting. This change is typically less than $20^{\circ}$ [9], although it is not yet entirely clear what degree of angular difference in these parameters indicates a stiff spine. When the pelvic tilt does not adequately increase, there is consequently less acetabular anteversion when in a sitting position and, therefore, increased risk of impingement and posterior dislocation.

Patient-specific acetabular component position can be decided based on these standing/ 
Fig. 13.2 Full leg-length standing anteroposterior (a) and lateral (b) and sitting anteroposterior (c) and lateral (d) films

obtained on long cassette

\section{a}

列

\section{b}

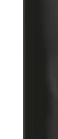

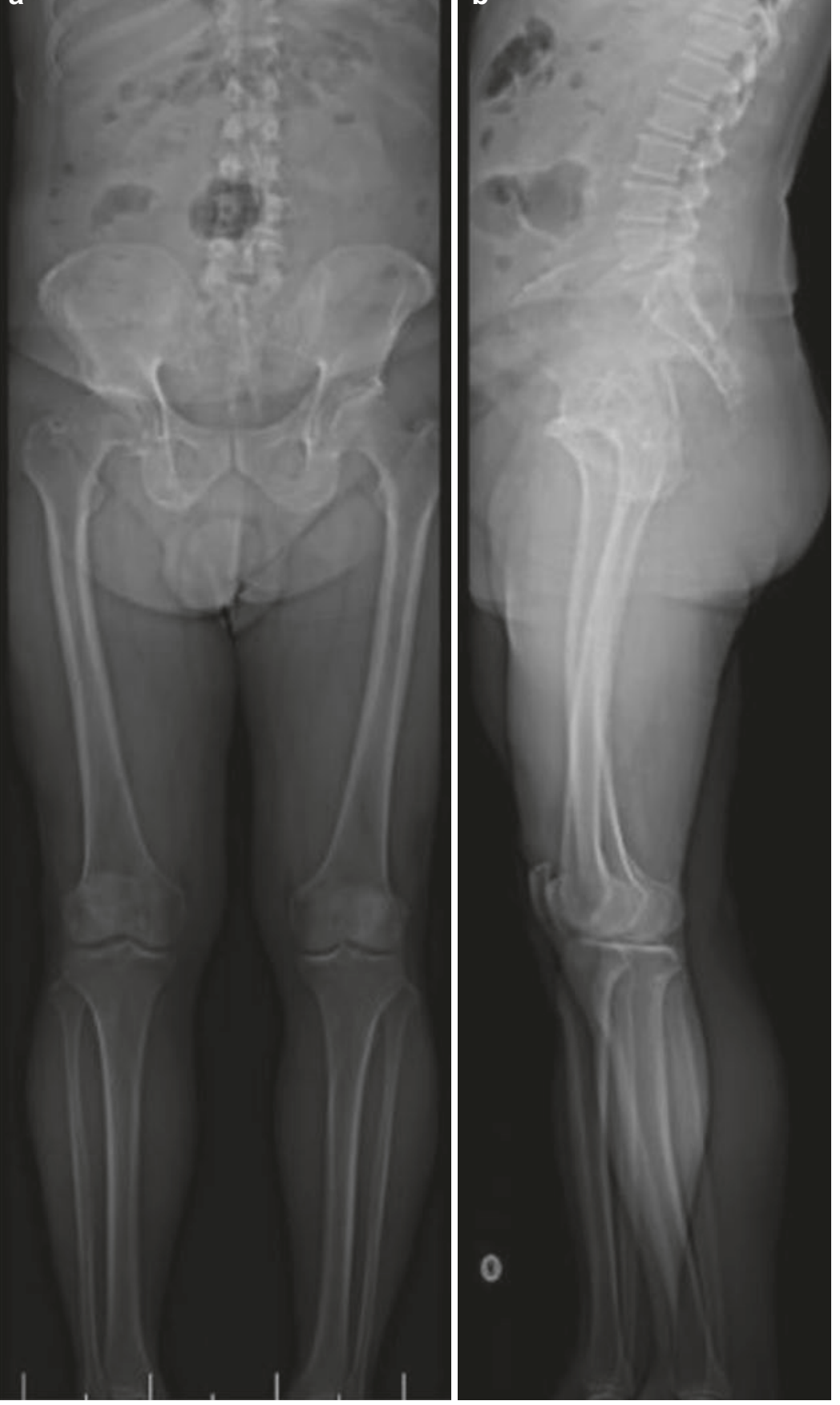


Fig. 13.2 (continued)
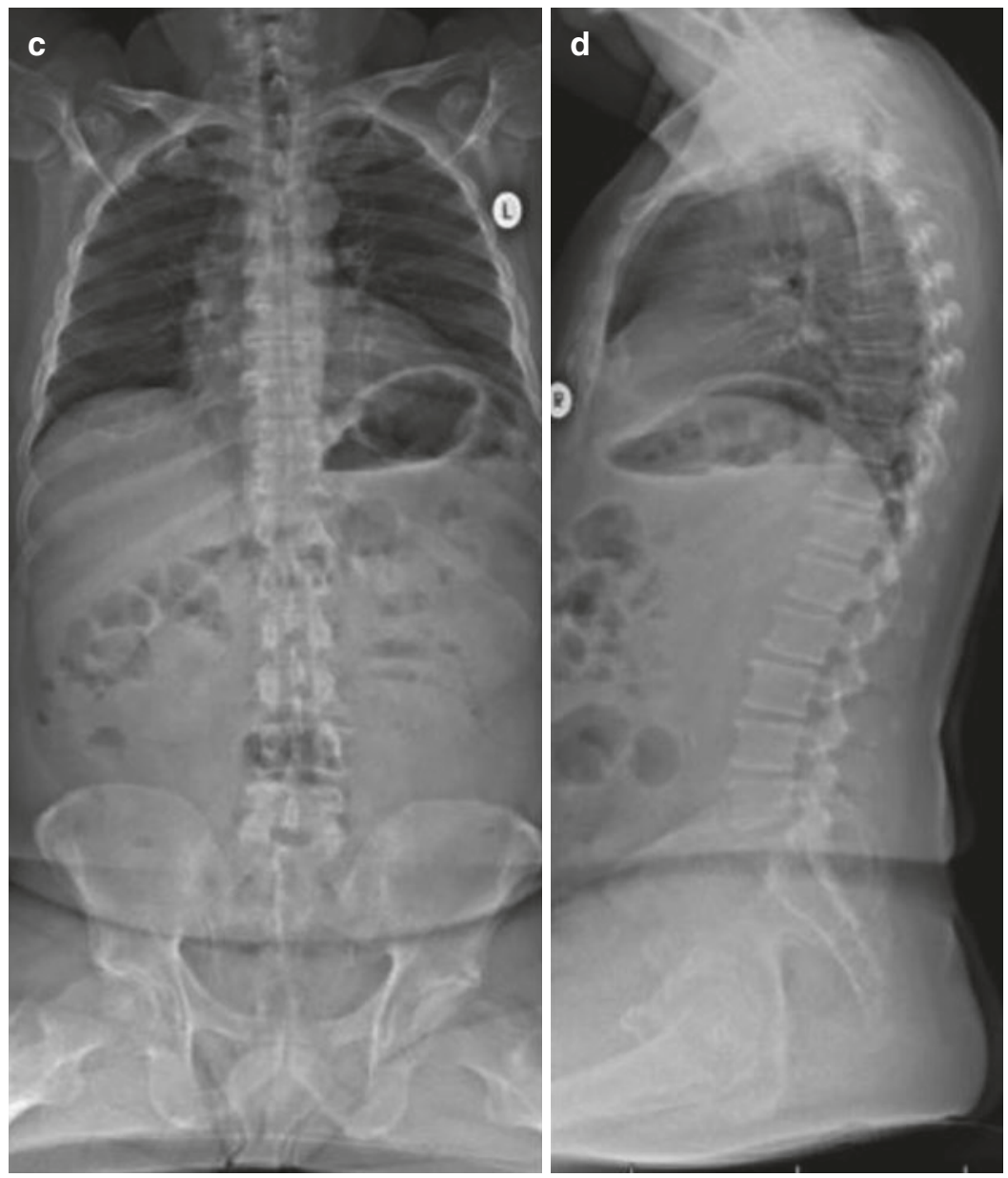

sitting alignment films and changes in spinopelvic parameters. Increasing cup anteversion may be warranted in patients with a significantly stiff lumbar spine and very limited changes in pelvic tilt from standing to sitting. In higher-risk cases, dual mobility implants may be considered (Fig. 13.4). Without obtaining this radiographic assessment of the patient's spino-pelvic dynamics, it is difficult to identify who may be at a higher risk of dislocation, and choosing the same target cup position for all may lead to dislocation in those with stiff or fused lumbar spines.

\subsubsection{Stepwise Evaluation of Acetabular Component Position in Total Hip Instability}

When evaluating a patient with prosthetic hip instability for revision surgery or a patient at high-risk of dislocation following primary THA, it is critical to employ a stepwise radiographic assessment of component positioning to determine the optimal patient-specific functional implant position that minimizes the risk of instability. 


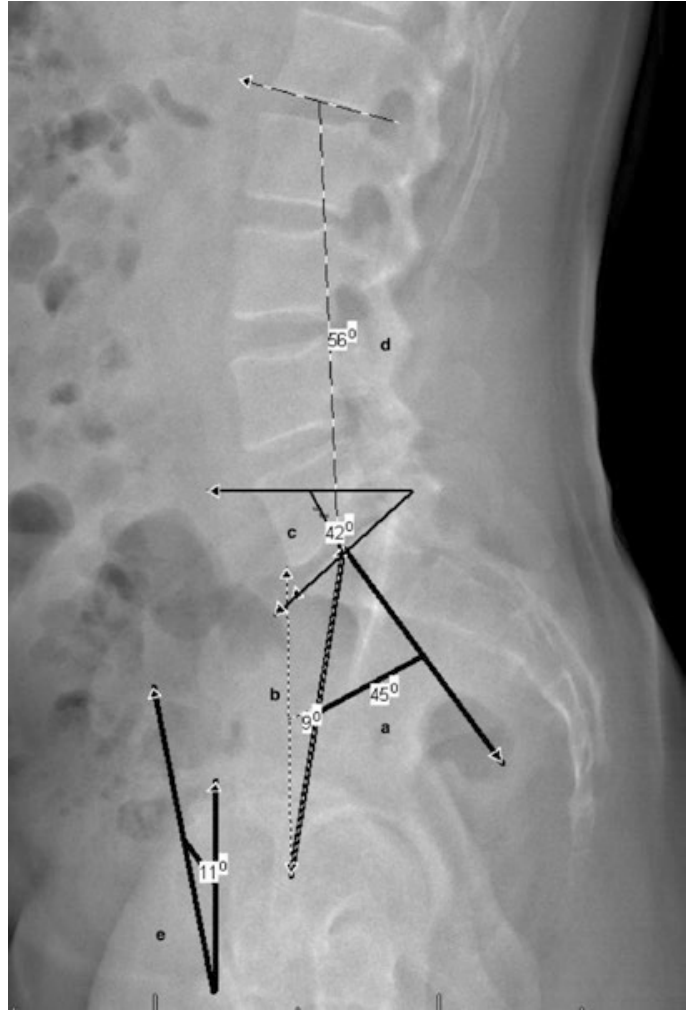

Fig. 13.3 Standing lateral radiograph demonstrating spino-pelvic parameter measurements. Pelvic incidence, a; pelvic tilt, b; sacral slope, c; lumbar lordosis, d; anterior pelvic plane, e
Initially a supine AP pelvis may be obtained, and the supine cup abduction and anteversion may be deduced as previously described. A standing or weight-bearing AP view of the pelvis can then be obtained for comparison with the supine view. This standing film offers an assessment of the cup abduction and anteversion in the patient's functional standing weight-bearing position. Pelvic obliquity, rotation, or tilt may affect the functional cup abduction or anteversion positions. For instance, patients with excessive anterior pelvic tilt will functionally have less cup anteversion in a standing position.

Subsequently, sitting and standing lateral fulllength radiographs may then be obtained. Lumbar degenerative processes including spinal fusion, spondylosis, spondylolisthesis, or sagittal spinal imbalance or deformity can be assessed through these images. These lumbar pathologies significantly affect spino-pelvic motion and therefore have consequences that impact acetabular component position and therefore risks of instability, prosthetic impingement, and edge loading. The spino-pelvic parameters listed above can be assessed from these sitting to standing films, and based on changes in these parameters, the change in cup anteversion between these two functional
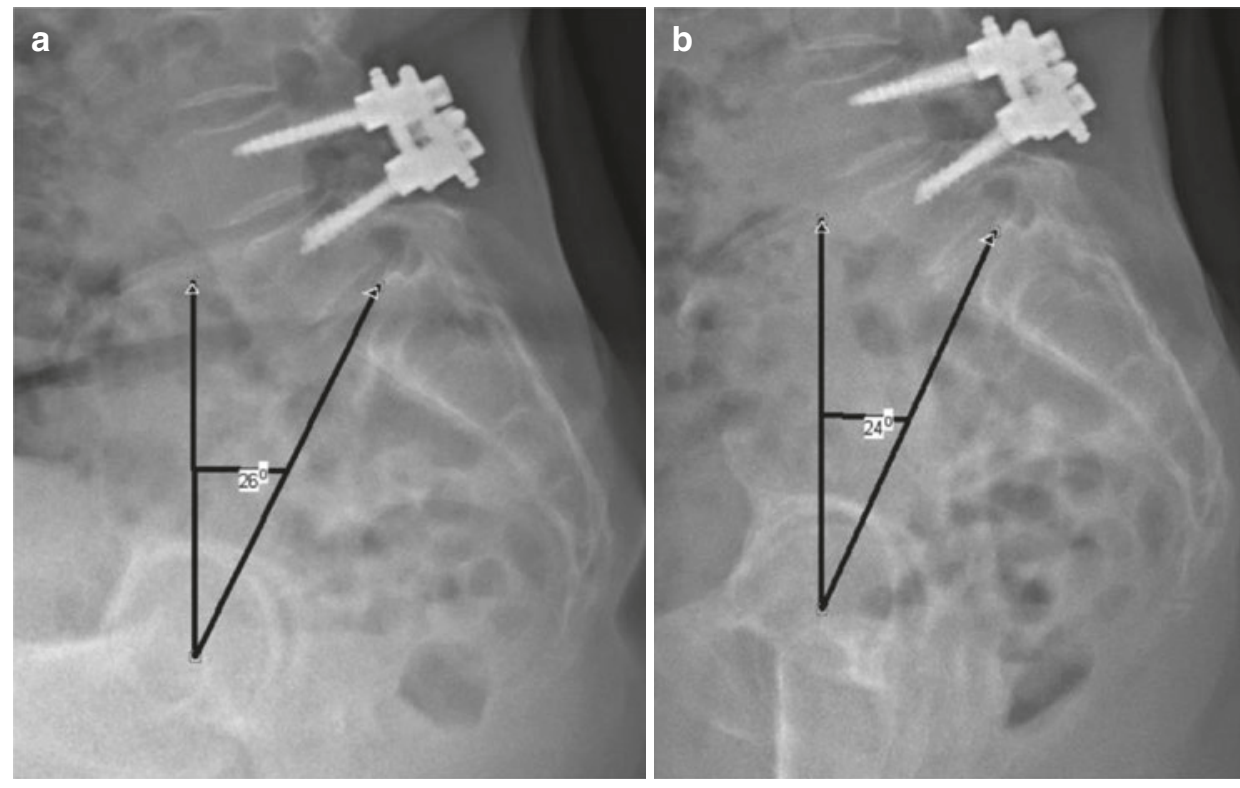

Fig. 13.4 Lateral sitting (a) and standing (b) plain films demonstrating minimal pelvic tilt change between the two functional positions in a patient with posterior L4-L5 spi- nal fusion for degenerative lumbar disease. The lack of pelvic tilt change limits cup anteversion in a sitting position, which increases the risk of dislocation 
positions may be deduced as described by Lembeck [13]. In cases with limited changes in pelvic tilt, and therefore limited increase in cup anteversion when going from standing to sitting, it may be important to consider increasing the anteversion of the revision acetabular component to account for this limited pelvic mobility.

\subsection{3-D Imaging to Assess Patient-Specific Component Position}

\subsubsection{Computed Tomography 3-D Imaging}

Obtaining a CT scan prior to THA is not routine practice but is often done as part of the protocol of some robotic-assisted computer navigation tools. CT imaging can be used to template component positioning preoperatively and offers the advantage of axial imaging of the acetabular anteversion, anterior and posterior wall thickness, and a better delineation of the proximal femoral anatomy including femoral version. In complex cases of osteolysis and revision THA, CT imaging can better delineate bone loss and becomes even more important for preoperative planning and implant choice. However, CT imaging is still a static imaging modality that does not consider the dynamic changes in acetabular orientation between different functional positions. Furthermore, CT imaging may be used to determine femoral component version, which is useful when evaluating total hip instability.

\subsubsection{Statistical Shape Modeling Method of Converting 2-D to 3-D Imaging}

Although three-dimensional imaging is useful in preoperative planning and templating for patientspecific component positioning in THA, it is often derived from CT or MRI imaging which carry the inherent disadvantages of being expensive, time-consuming, and may expose the patient to significant ionizing radiation (CT).

A statistical shape model (SSM) reconstruction technique has been used to create a patient-specific
3-D surface model of the pelvis based on a single 2-D AP view of the pelvis [14]. This technique is predicated on landmark-based initialization and iterative matching of apparent image contours extracted from the 2-D radiograph to create a 3-D reconstruction. This method is a feasible technique to create patient-specific 3-D images, which may be used for preoperative planning without obtaining MRI or CT scan. This technique has also been successful in creating 3-D reconstructions of the lumbar vertebral anatomy [15].

\subsubsection{The Use of CT Imaging in Assessment of Personalized Component Implantation}

Precise assessment of conventionally implanted hip prostheses is possible with CT imaging by measuring component orientation relative to anatomical landmarks. For example, cup orientation and prosthetic neck anteversion are respectively measured relatively to the anterior pelvic plane and posterior condylar line. Similarly, CT imaging is useful in accurate assessment and quality control of personalized THA implantation, particularly if preoperative CT imaging is available for comparison (osteoarthritic vs. prosthetic anatomy). Comparisons of the pre- and postoperative imaging can indicate whether the native proximal femoral and acetabular orientations and the hip center of rotation have been appropriately reproduced and whether the components were implanted with accuracy compared to the preoperative template (Fig. 13.5). 3-D CT imaging of the native hip or the planned hip replacement and the executed THA can be overlaid to provide insight of the precision of the personalized implantation technique. If pre-operative 3-D imaging is unavailable, a direct comparison between the prosthetic and contralateral hip may be of utility. Nevertheless, this method is may be limited, as the symmetry index between the axial anatomical parameters (femoral neck and acetabular anteversion) of both hips in a given individual may be weaker than previously thought. Despite this utility in the postoperative evaluation of a personalized THA, CT imaging is a static modality, obtained in a supine position, and is best interpreted in conjunction with the previ- 

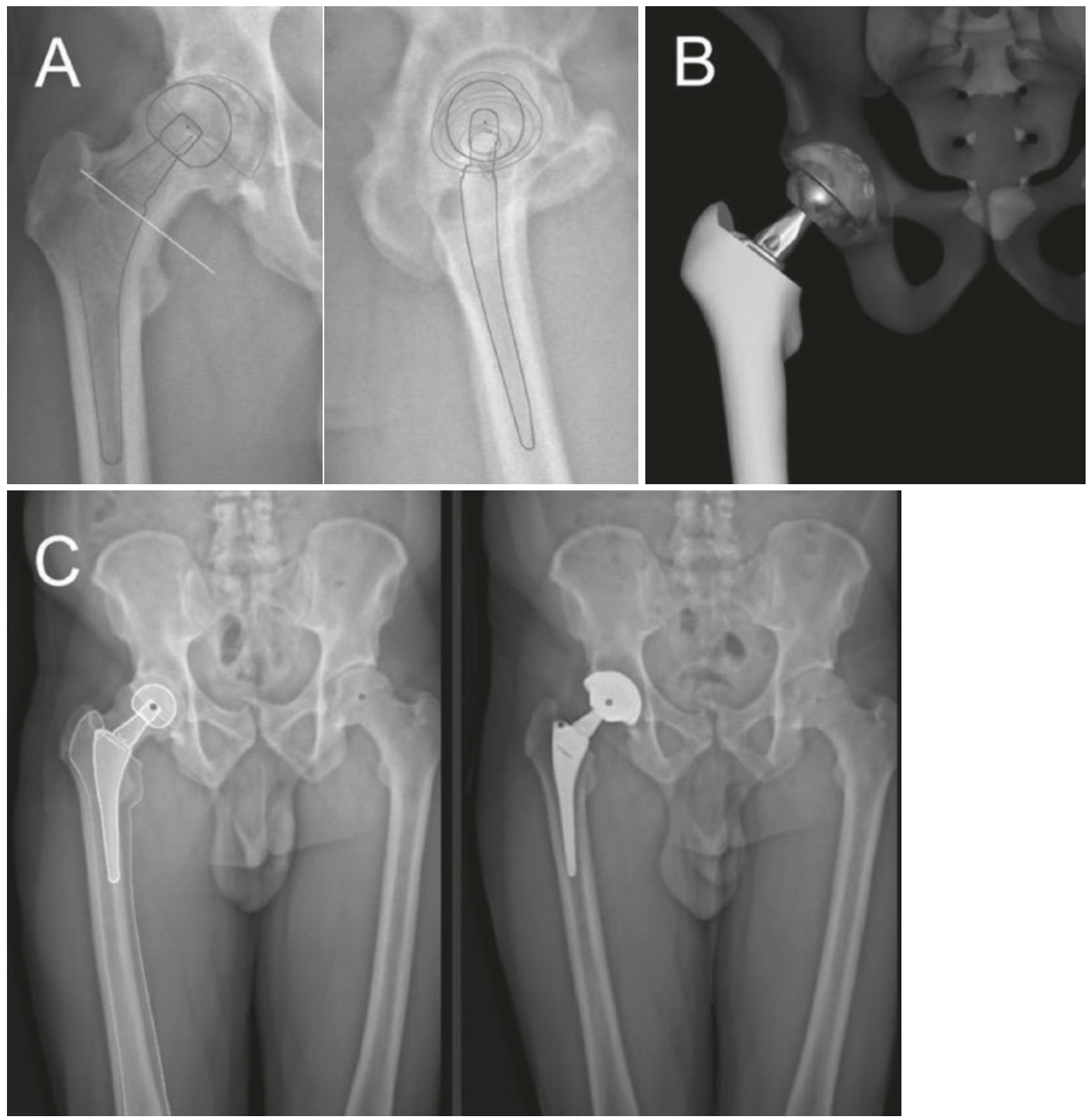

Fig. 13.5 This figure illustrates the planning of a total hip replacement on bi-dimensional EOS images (a), with tri-dimensional rendering (b) and relocation of postopera-

ously mentioned dynamic radiographs assessing spino-pelvic dynamics for a given patient.

\subsection{Conclusion}

Traditional plain radiography in the form of an AP pelvis and frog or cross table lateral of the hip are useful but may not capture spino-pelvic tive pelvic radiograph (c) (With the courtesy and permission of E. Maury, MD, University Hospital of Montpellier, France)

dynamics, which are critical to stability of THA. Based on recent findings, the concept of a defined "safe zone" of component position has evolved to a more dynamic and functional definition. In order to determine this appropriate patient-specific "safe zone," modern imaging techniques such as sitting and standing alignment plain radiographs are necessary for improved understanding of spino-pelvic dynamics and 
more appropriate component positioning to minimize the risk of instability and maximize bearing performance in THA. Personalized total hip component implantation should aim to recreate normal hip joint anatomy, with a "safe zone" that matches an individual's spino-pelvic dynamics. Three-dimensional imaging systems can be useful in assessing the accuracy and quality of personalized hip implantation.

\subsection{Case Presentation}

A 75-year-old man with a history of lumbar radiculopathy initially underwent a primary right THA in 2014. He subsequently suffered two separate incidents of anterior right THA dislocation 4 years later, both in a position of hip extension. Preoperative evaluation of his total hip instability comprised of a supine AP pelvis, cross table lateral of the right hip, as well as sitting and standing AP and lateral alignment films (Fig. 13.6). Comparison of pelvic tilt from standing to sitting positions demonstrated limited change, signifying a stiff lumbar spine. Furthermore, in a standing position, the cup anteversion was found to be approximately $35^{\circ}$, while cup abduction was approximately $50^{\circ}$ with respect to the coronal plane. Given this cup malposition, he was indicated for an acetabular component revision. Intraoperatively, stem version was found to be appropriate, and the stem was retained. However, the cup was revised to a dual mobility acetabular component, using computer navigation to place the new component in a position of less anteversion and inclination. Postoperatively, he recovered well, without further episodes of instability at 6 months of follow-up.
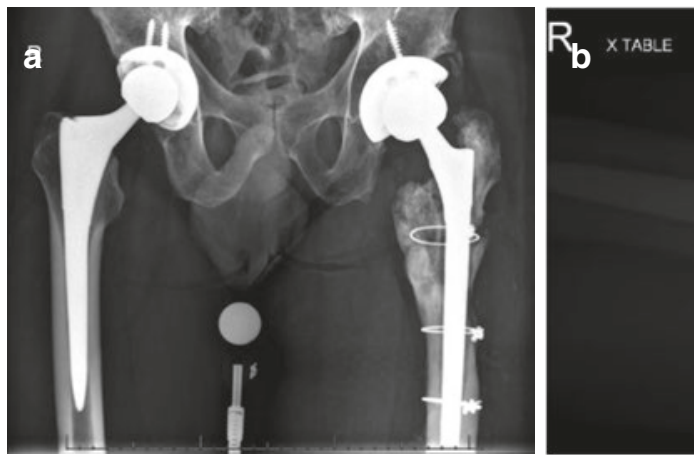

Fig. 13.6 Preoperative radiographic evaluation of a right total hip arthroplasty with anterior instability in the setting of degenerative lumbar stiffness. (a) Supine AP pelvis. (b) Supine cross table lateral view demonstrating the acetabular component anteversion measuring $48^{\circ}$ using Woo and Morrey's method and $31^{\circ}$ using the ischio-lateral method. This discrepancy can be attributed to this increased patient's tilt in a supine position. (c) AP and lateral sitting and standing alignment films were obtained. (d) Using software analysis (Intellijoint) of the sitting and standing alignment films, the anterior pelvic plane-pelvic tilt angle change from standing to sitting is noted to be limited, indicating stiffness in lumbar spino-pelvic mobility. Additionally, the acetabular component inclination and anteversion in the standing position were noted to be 51 and $35^{\circ}$, respectively 
Fig.13.6 (continued)

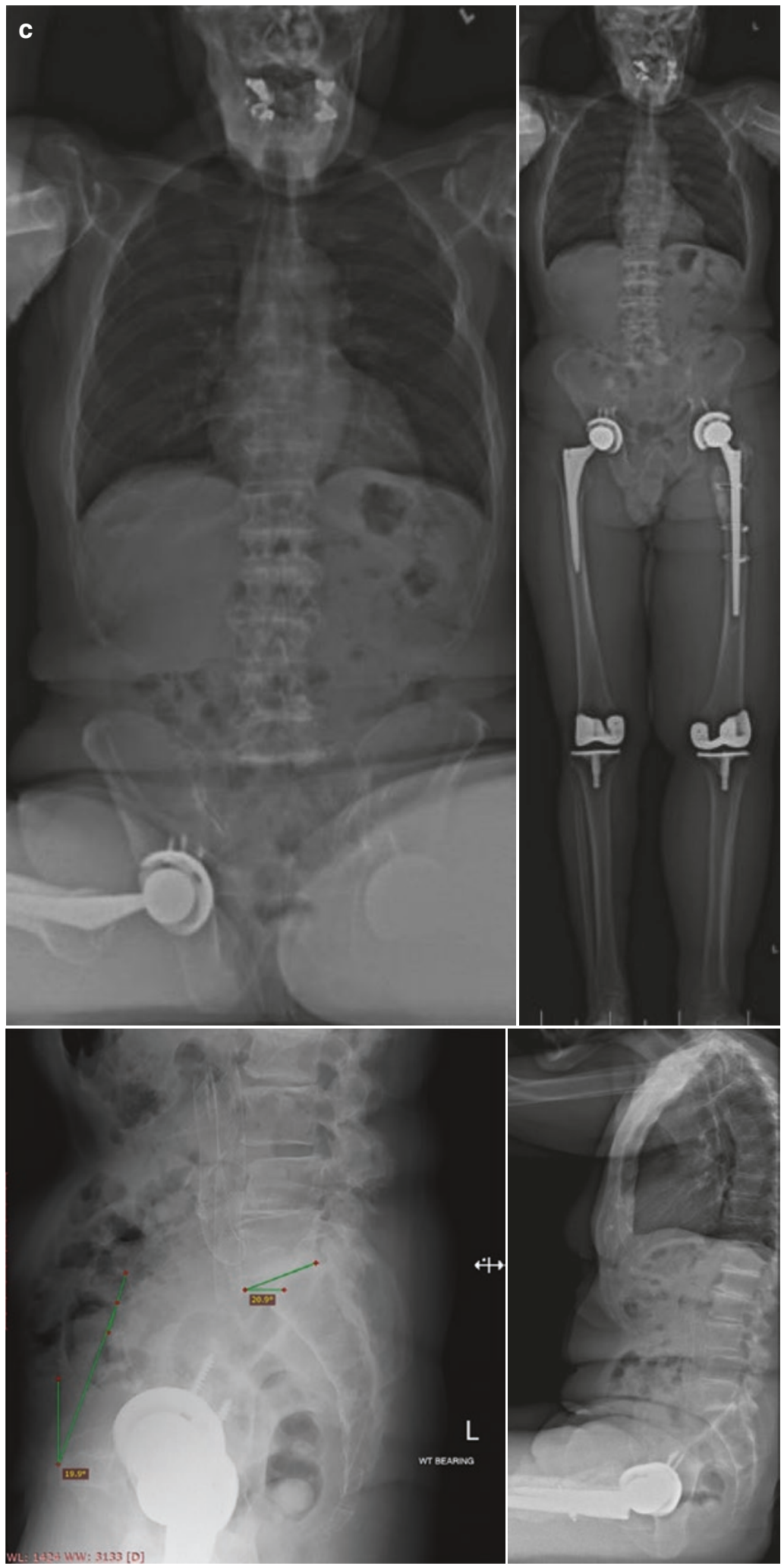




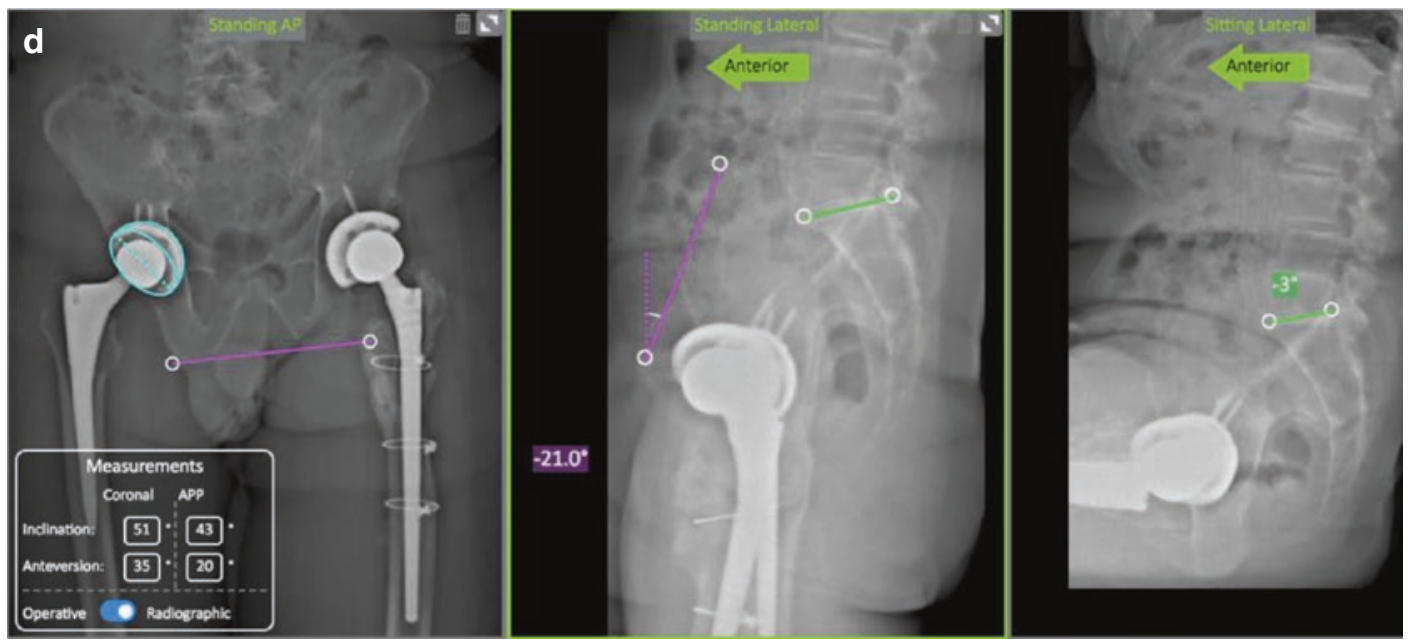

Fig. 13.6 (continued)

\section{References}

1. Lewinnek GE, Lewis JL, Tarr R, Compere CL, Zimmerman JR. Dislocations after total hipreplacement arthroplasties. J Bone Joint Surg Am. 1978;60(2):217.

2. Weber M, Lechler P, von Kunow F, Vollner F, Keshmiri A, Hapfelmeier A, Grifka J, Renkawitz T. The validity of a novel radiological method for measuring femoral stem version on anteroposterior radiographs of the hip after total hip arthroplasty. The Bone Joint J. 2015;97-B(3):306.

3. Woerner ML, Weber M, Craiovan BS, Springorum HR, Grifka J, Renkawitz TF. Radiographic assessment of femoral stem torsion in total hip arthroplasty-a comparison of a caput-collum-diaphyseal anglebased technique with the budin view. J Arthroplast. 2016;31(5):1117.

4. Pulos N, Tiberi Iii JV 3rd, Schmalzried TP. Measuring acetabular component position on lateral radiographs-ischio-lateral method. Bull NYU Hosp Jt Dis. 2011;69(Suppl 1):S84.

5. Abdel MP, von Roth P, Jennings MT, Hanssen AD, Pagnano MW. What safe zone? The vast majority of dislocated THAs are within the Lewinnek safe zone for acetabular component position. Clin Orthop Relat Res. 2016;474(2):386.

6. Buckland AJ, Puvanesarajah V, Vigdorchik J, Schwarzkopf R, Jain A, Klineberg EO, Hart RA, Callaghan JJ, Hassanzadeh H. Dislocation of a primary total hip arthroplasty is more common in patients with a lumbar spinal fusion. The Bone Joint J. 2017;99-B(5):585.

7. DelSole EM, Vigdorchik JM, Schwarzkopf R, Errico TJ, Buckland AJ. Total hip arthroplasty in the spinal deformity population: does degree of sagittal deformity affect rates of safe zone placement, instability, or revision? J Arthroplast. 2017;32(6):1910.

8. Fujita K, Kabata T, Maeda T, Kajino Y, Iwai S, Kuroda $\mathrm{K}$, Hasegawa K, Tsuchiya H. The use of the transverse acetabular ligament in total hip replacement: an analysis of the orientation of the trial acetabular component using a navigation system. Bone Joint J. 2014;96-B(3):306.

9. Kanawade V, Dorr LD, Wan Z. Predictability of acetabular component angular change with postural shift from standing to sitting position. J Bone Joint Surg Am. 2014;96(12):978.

10. Stefl M, Lundergan W, Heckmann N, McKnight B, Ike H, Murgai R, Dorr LD. Spinopelvic mobility and acetabular component position for total hip arthroplasty. The Bone Joint J. 2017;99-B(1 Suppl A):37.

11. Esposito CI, Carroll KM, Sculco PK, Padgett DE, Jerabek SA, Mayman DJ. Total hip arthroplasty patients with fixed spinopelvic alignment are at higher risk of hip dislocation. J Arthroplast. 2018;33(5):1449.

12. Pierrepont JSC, Miles B, O'Connor P, Ellis A, Molnar R, Baré J, Solomon M, McMahon S, Shimmin A, Li Q, Walter L, Marel E. Patient-specific component alignment in total hip Arthroplasty. Reconstr Review. 2016;6(4):27.

13. Lembeck B, Mueller O, Reize P, Wuelker N. Pelvic tilt makes acetabular cup navigation inaccurate. Acta Orthop. 2005;76(4):517.

14. Zheng G. Statistical shape model-based reconstruction of a scaled, patient-specific surface model of the pelvis from a single standard AP X-ray radiograph. Med Phys. 2010;37(4):1424.

15. Zheng G, Nolte LP, Ferguson SJ. Scaled, patientspecific 3D vertebral model reconstruction based on 2-D lateral fluoroscopy. Int J Comput Assist Radiol Surg. 2011;6(3):351. 
Open Access This chapter is licensed under the terms of the Creative Commons Attribution 4.0 International License (http://creativecommons.org/licenses/ by/4.0/), which permits use, sharing, adaptation, distribution and reproduction in any medium or format, as long as you give appropriate credit to the original author(s) and the source, provide a link to the Creative Commons license and indicate if changes were made.
The images or other third party material in this chapter are included in the chapter's Creative Commons license, unless indicated otherwise in a credit line to the material. If material is not included in the chapter's Creative Commons license and your intended use is not permitted by statutory regulation or exceeds the permitted use, you will need to obtain permission directly from the copyright holder. 